\title{
Parameter identification in particle models
}

\author{
H. F. P. van den Boogaard', M. J. J. Hoogkamer', and A. W. Heemink ${ }^{2}$ \\ ${ }^{1}$ Delft Hydraulics, POB 177, NL-2600 MH, Delft, The Netherlands \\ ${ }^{2}$ Delft Univ. Technology, Dept. of Applied Analysis, POB 356, NL-2628AJ, Delft, \\ The Netherlands
}

\begin{abstract}
For the simulation of the transport of dissolved matter particle models can be used. In this paper a technique is developed for the identification of uncertain parameters in these models. This model calibration is formulated as an optimization problem and is solved with a gradient based algorithm. Here adjoint particle tracks are used for the calculation of the gradient of the cost function. The performance of the calibration method is illustrated by simulations and an application to a river Rhine water quality calamity in November 1986.
\end{abstract}

Key words: Particle models, transport equations, parameter identification, adjoint modelling, cost function, gradient.

\section{Introduction}

Nowadays it is common practice to use mathematical models for the simulation of water flow and the transport of dissolved matter in large scale water systems as rivers, seas or estuaries. The dissolved matter can be salt or pollutants.

Usually these mathematical models have the form of one or more coupled partial differential equations (PDE). These PDE describe the evolution of the relevant physical processes (water levels, water velocities, or the concentrations of the dissolved matter) in the water system. The well known conservation laws of mass, impulse and/or energy form the basis of these state equations.

It is usually not possible to solve the state equations analytically and as a consequence it must be relied on numerical methods. Well known are the so-called finite difference methods (FDM). In this approach the temporal and spatial coordinates are discretized and the PDE are approximated in discrete form with a numeric scheme. Recently an alternative for the numerical simulation of transport equations has obtained much attention. This alternative method is based on so-called particle models (also known as random walk models). In contrast to FDM solvers that simulate the spreading of dissolved matter by means of concentrations, a particle model calculates the track of many individual particles. From these tracks a concentration of the dissolvent can again be derived.

There are some clear advantages of particle models over FDM methods for the simulation of transport of dissolved matter:

(i) Particle models are strictly mass conserving (unless mass decay is explicitly modelled, see Section 3).

(ii) Particle models can deal with arbitrarily steep concentration gradients. 
(iii) Particle models do not require a spatial grid for the calculation of the transport of the dissolved matter; it is thus possible to model processes on an arbitrarily small scale (subgrid processes, influence of turbulence, ....etc.).

Particle models are thus well suited for the simulation of 'near field' transport processes, i.e. in case the dissolved matter has a pronounced, peaked spatial distribution.

On the other hand particle models will not be suited for the simulation of large scale transport processes (e.g. salt intrusion in estuaries). Such simulations would require very many particles for an appropriate representation of the concentration.

Since numerical models can be used for the description and prediction of the state of water systems, they are an important tool for the management and control of these systems. For such decision making problems the numerical model must provide an accurate description of the water system for a wide range of conditions. Although numerical models usually have a sound physical basis, their accuracy is often limited. The reason of these shortcomings are uncertainties in one or more model coefficients. Well known examples are friction coefficients in water flow models and dispersion coefficients in transport models. To improve the performance of the model it is common use to adjust these coefficients until the agreement of model and measurements is satisfactory. This so-called calibration of the numerical model is often a tedious problem.

In recent years significant progress has been made in the development of objective, automized and efficient calibration techniques for FDM numerical models. As examples the Kalman Filter (Heemink, 1986) and optimal control methods in combination with adjoint modelling (Chavent, 1980; Merckx 1987; van den Boogaard, 1988) can be mentioned. At the moment, no structured techniques seem to be available for particle models. In this paper we develop a method for the identification of parameters in particle models that is based on adjoint modelling.

\section{Particle models}

A particle model is a description of a transport process by means of random walk models. Whereas transport models are usually deterministic, a particle model is a stochastic model. In a particle model the track of many individual particles is calculated using a random generator. The ensemble of all these tracks describes the spread of a solute in the water system. In this section a brief summary of the relevant theory is given. Here and in the remainder of this paper we will restrict ourselves to particle models in one (spatial) dimension. For applications of particle models in two dimensions, see e.g. Heemink (1990) and Uffink (1990).

The Langevin fluctuation equation forms the basis of particle models. This equation is an example of a stochastic differential equation. It describes the particle's position as function of time and it has the following form:

$d \mathbf{X}(t)=v(\mathbf{X}, t) d t+D(X, t) d W(t)$

In this stochastic differential equation,

$\mathbf{X}(\cdot) \quad: \quad$ The random position of a particle as function of time.

$\mathrm{v}(\because$,$) \quad : The drift coefficient, \mathrm{v}(\cdot, \cdot)$ is a deterministic function.

$\mathrm{D}(\because)$ : A coefficient related to the diffusion coefficient; $\mathrm{D}(\because)$ is a deterministic function.

$\mathbf{W}(\cdot) \quad$ : A Wiener-Levy process; $\mathbf{W}(\cdot)$ is a random function of time (stochastic process). 
Equation (1) will be interpreted according to Ito's rule (see e.g. van Kampen, 1981b).

The Wiener-Levy process $\mathbf{W}(\cdot)$ is as well known as a Brownian motion. It is a Gaussian and Markovian process characterized by:

$E[W(t)]=0$

$E\left[W\left(t_{1}\right) \cdot W\left(t_{2}\right)\right]=\operatorname{Min}\left(t_{1}, t_{2}\right)$.

W( $)$ is thus a nonstationary process. Since it is Gaussian equation (2) provides a complete characterization of the process. We mention some other important properties of $\mathbf{W}(\cdot)$. The Wiener-Levy process is an independent increment process, i.e. the increments $W\left(t_{4}\right)-W\left(t_{3}\right)$ and $W\left(t_{2}\right)-W\left(t_{1}\right)$ are independent for $t_{4}>t_{3}>t_{2}>t_{1}$. Each increment $W\left(t_{2}\right)-W\left(t_{1}\right)$ is Gaussian distributed with mean 0 and variance $t_{2}-t_{1}$.

At this moment the question may arise what the relation is between the random walk model of equation (1) on one hand, and the transport equations that are used in water quality studies on the other hand. We will deal with this problem in Section 3.

\section{The relation of particle models and transport models}

In this section the relation between random walks and transport equations is discussed. For this purpose the following two functions are compared:

(i) The probability density function $\mathbf{f}(* ;)$ of a particle's position $\mathbf{X}(\cdot)$, where $\mathbf{X}(\cdot)$ satisfies the stochastic differential equation (1).

(ii) The function $\mathrm{c}(\cdot, ;)$ that describes the time evolution of the concentration of dissolved matter.

It will be shown here that $f(\because)$ and $c(;)$ are related as follows:

$f(x, t)=A(x, t) \cdot c(x, t)$

where:

$c(x, t)$ is the concentration of the dissolved matter at $(x, t)$,

$A(x, t)$ is the cross-section (of the river or estuary).

The probability density function $f(\cdot ;)$ of a particle's position at time $t$ is defined by,

$f(x, t):=\lim _{\Delta x \rightarrow 0} \frac{p[x \leq X(t)<x+\Delta x]}{\Delta x}$

By using statistical techniques it can be shown that the probability density function $\mathrm{f}(\cdot ;)$ satisfies a diffusion equation, the so-called Fokker-Planck equation. The Fokker-Planck equation associated with the random walk of equation (1) is:

$\frac{\partial}{\partial t} f(x, t)=-\frac{\partial}{\partial x}[v(x, t) f(x, t)]+\frac{1}{2} \frac{\partial^{2}}{\partial x^{2}}\left[D^{2}(x, t) f(x, t)\right]$.

For details and further explanation the reader is referred to Middleton (1960), van Kampen (1981a), and Stratonovich (1963).

Transport equations in one space dimension have usually the form of an advectiondispersion equation: 
$\frac{\partial}{\partial t}[A(x, t) c(x, t)]=-\frac{\partial}{\partial x}[Q(x, t) c(x, t)]+\frac{\partial}{\partial x}\left[E(x, t) A(x, t) \frac{\partial}{\partial x} c(x, t)\right]$.

In this advection-dispersion equation:

$Q(x, t)$ is the discharge (through the cross section $A(x, t)$ )

$E(x, t)$ is the dispersion coefficient.

From $\mathrm{A}(\because)$ and $\mathrm{Q}(\because)$ the velocity $\mathrm{u}(\mathrm{x}, \mathrm{t})$ (averaged over the cross-section) can be found as follows:

$\mathbf{u}(\mathbf{x}, \mathbf{t})=\frac{\mathbf{Q}(\mathbf{x}, \mathbf{t})}{\mathbf{A}(\mathbf{x}, \mathbf{t})}$.

Clearly equation (5) and equation (6) are much alike. It must yet be verified how the functions $\mathrm{f}(\because), \mathrm{v}(, ;)$ and $\mathrm{D}(\because)$ in equation $(5)$ on one hand, and the functions $\mathrm{Q}(\because), \mathrm{A}(, ;)$ $\mathrm{E}(\cdot$,$) and \mathrm{c}(\cdot$,$) in equation (6)$ on the other hand, are related. This can be done as follows. First it is noted that the concentration $c(x, t)$ (mass per unit of volume) is related to the mass density $\mu(x, t)$ (mass per unit of length) according to:

$\mu(x, t)=A(x, t) c(x, t)$.

Substitution of equation (8) into equation (6) results in the following partial differential equation for $\mu(\because)$ :

$\frac{\partial}{\partial t} \mu(x, t)=-\frac{\partial}{\partial x}\left[\left\{u(x, t)+\frac{E(x, t) \frac{\partial}{\partial x} A(x, t)}{A(x, t)}\right\} \mu(x, t)\right]+\frac{\partial}{\partial x}\left[E(x, t) \frac{\partial}{\partial x} \mu(x, t)\right]$.

This equation can be rewritten into a Fokker-Planck equation:

$$
\frac{\partial}{\partial t} \mu(x, t)=-\frac{\partial}{\partial x}\left[\left\{u(x, t)+\frac{1}{A(x, t)} \frac{\partial}{\partial x}[E(x, t) A(x, t)]\right\} \mu(x, t)\right]+\frac{1}{2} \frac{\partial^{2}}{\partial x^{2}}[2 E(x, t) \mu(x, t)] .
$$

As a result of equations $(10),(5)$ and $(8) f(x, t)=\mu(x, t)=A(x, t) \cdot c(x, t)$ and the following random walk model can be used for the simulation of the transport model of equation (6):

$d X(t)=v(X, t) d t+D(X, t) d W(t)$

where 


$$
\begin{aligned}
v(x, t) & =u(x, t)+\frac{1}{A(x, t)} \frac{\partial}{\partial x}[E(x, t) A(x, t)] \\
& =\frac{Q(x, t)+\frac{\partial}{\partial x}[E(x, t) A(x, t)]}{A(x, t)} \\
& =\frac{Q(x, t)}{A(x, t)}+\frac{\partial E(x, t)}{\partial x}+E(x, t) \frac{\frac{\partial A(x, t)}{\partial x}}{A(x, t)}
\end{aligned}
$$

and

$D(x, t)=\sqrt{2 E(x, t)}$

Note that the drift term $v(\cdot ;)$ in the fluctuation equation is not simply the water velocity $u(\because)$ although they are closely related. The difference is introduced by the spatial variability of the cross section and the dispersion coefficient.

So far the transport of a conservative dissolvent has been considered. In water quality problems the suspended matter is often subjected to decay due to chemical or biological interactions. The present particle model can easily be extended to include (exponential) decay as well: apart from the fluctuation equation (11) the following equation for the time evolution of the mass of a particle is proposed,

$$
\begin{aligned}
& \mathbf{d} \mathbf{M}(t)=-\gamma(\mathbf{X}, t) \mathbf{M}(t) d t \\
& \mathbf{M}\left(t_{0}\right)=\mu_{0}
\end{aligned}
$$

where,

$\mu_{0}$ is the initial mass of a particle,

$\mathbf{M}(\cdot)$ is the particle's mass at time $t$, and

$\gamma(\cdot)$ is the decay coefficient.

The decay coefficient $\gamma(\because)$ is a deterministic function (although its argument is stochastic) and it can depend on both the spatial and time coordinate. As a consequence the particle's loss of mass depends on its trajectory. Equation (13) has been chosen because of the fact that the corresponding equation for the concentration $c(x, t)$ is the following well-known, nonconservative, transport equation (see Appendix B):

$$
\frac{\partial}{\partial t}[A(x, t) c(x, t)]=-\frac{\partial}{\partial x}[Q(x, t) c(x, t)]+\frac{\partial}{\partial x}\left[E(x, t) A(x, t) \frac{\partial}{\partial x} c(x, t)\right]-\gamma(x, t) A(x, t) c(x, t)
$$

\section{The calibration of adjustable parameters in particle models}

Before the particle model of equations (11-14) can be applied, an appropriate choice for the uncertain coefficients must be made. In Section 1 it was argued that in numerical models coefficients are often not known accurately and must be determined by calibration. In this section, model calibration is described as an optimization process. 


\subsection{The parameterization of adjustable coefficients}

In transport models, the following coefficients will in general not be known: the dispersion coefficient governing the spread of the dissolved matter, the (initial) mass of the solute, and the decay coefficient.

In practice these coefficients are usually adapted by "trial and error" until the model outcome compares well with measurements. Since the coefficients can depend on both the temporal and spatial coordinates a huge number of quantities have to be adjusted. On the other hand only a very limited number of measurements is usually available. To overcome this mismatch the adjustable coefficients are parameterized. The adjustable parameters in the parameterization of the dispersion coefficient and the decay coefficient are denoted by $\alpha:=$ $\left(\alpha_{1}, \alpha_{2}, \ldots, \alpha_{A}\right)^{\mathrm{T}}$ and $\beta=\left(\beta_{1}, \beta_{2}, \ldots, \beta_{B}\right)^{\mathrm{T}}$ respectively. For the initial mass of a particle only one parameter is required. It is denoted by $\mu_{0}$.

The parameterized dispersion coefficient and the decay coefficient are now written as,

$$
\begin{aligned}
& E(x, t)=E(x, t \mid \alpha) \\
& \gamma(x, t)=\gamma(x, t \mid \beta) .
\end{aligned}
$$

Possible parameterizations are given in section 6.

\subsection{The cost function}

Given a set of observations $\left\{\hat{c}\left(x_{m}, t_{m}\right) \mid m=1,2, \ldots M\right.$ of the concentrations of the dissolved matter, and $K$ particle tracks of the random walk model of equations (11-13), the agreement of the model and the observations can be formalized by a so called cost function. Here a quadratic criterion is used for the cost function:

$$
J=\sum_{m=1}^{M}\left\{c\left(x_{m}, t_{m}\right)-\hat{c}\left(x_{m}, t_{m}\right)\right\}^{2} \cdot \Psi_{m} .
$$

In this formula $c\left(x_{m}, t_{m}\right)$ is the model counterpart of $\hat{c}\left(x_{m}, t_{m}\right) \cdot \Psi_{m}$ is a nonnegative number that assigns a weight to the measurement $\hat{c}\left(x_{m}, t_{m}\right)$. The more accurate or significant the measurement is expected to be, the larger this weight is chosen. In case little is known about the accuracy of the measurements, the weights are chosen to be 1.0.

Note that the smaller the cost function $J$, the better the agreement of model and observations. In this way, calibration of the model has been translated into minimization of the cost function.

With regard to the model, the cost function is expressed in concentrations rather than particle tracks. The particle tracks $\left\{X_{k}(t) \mid k=1,2, \ldots, K\right\}$ can however be translated into a concentration $\mathrm{c}(\mathrm{x}, \mathrm{t})$ according to:

$$
c(x, t)=\frac{1}{A(x, t)} \sum_{k=1}^{K} M_{k}(t) \phi\left(x-X_{k}(t), t\right) \text {. }
$$

In this formula $M_{k}(t)$ is the mass of the $k$-th particle and $\phi(\cdot ;)$ is a point spread function. Introducing the integrated decay coefficient for the $k$-th particle determined by: 
$\Gamma_{\mathbf{k}}(\mathrm{t}):=\int_{b}^{t} \gamma\left(\mathrm{X}_{\mathbf{k}}(\mathrm{s}), \mathrm{s}\right) \mathrm{ds}$.

Equation (17) can be rewritten to:

$c(x, t)=\frac{\mu_{0}}{A(x, t)} \sum_{k=1}^{x} e^{-r_{k}(t)} \phi\left(x-X_{k}(t), t\right)$

See Appendix A for more details on the derivation of these equations.

\subsection{Minimization of the cost function}

Given the parameterization of the adjustable model coefficients, the calibration of the transport model is transformed into a minimization problem:

$\min _{\alpha, \hat{\beta}, \mu_{0}} J(c, \hat{c})$

Unfortunately the cost function depends implicitly on the parameters. This dependency is rather deeply nested: the chain of dependencies is given by

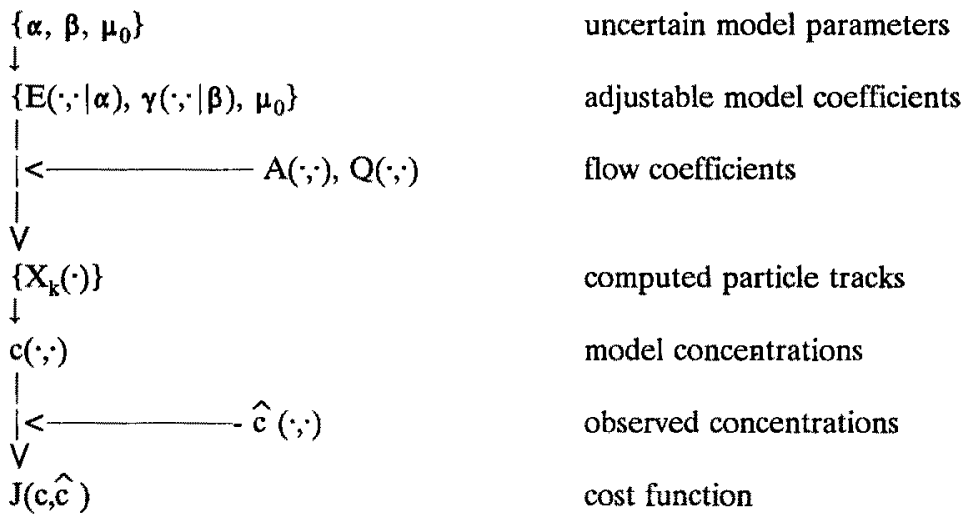

It is therefore not possible to find the minimum of $J$ analytically and the problem has to be solved numerically. Here a gradient descent method will be used (BFGS quasi-Newton method, see e.g. Press et al., 1989). The adjustable parameters are thus estimated iteratively: an initial guess of the parameters is repeatedly updated until the cost function is minimized.

The main problem is the calculation of the gradients $\nabla_{\alpha} \mathrm{J}, \nabla_{\beta} \mathrm{J}$ and $\partial \mathrm{J} / \partial \mu_{0}$. A straightforward method would be numerical differentiation, This means that the cost function $J$ is evaluated for the unperturbed parameter vector $\left(\alpha_{1}, \alpha_{2}, \ldots, \alpha_{A} ; \beta_{1}, \beta_{2}, \ldots, \beta_{B} ; \mu_{0}\right)$ and for each, components wise perturbation of this vector. The difference of these cost functions divided by the perturbation provides an approximation of the derivative of the cost function with respect to this component.

This method is computationally very expensive, especially if the number of adjustable parameters is large. From optimal control theory it is known that the gradient can be calculated extremely efficient by means of an adjoint system. This approach will be followed 
here as well. As a consequence an adjoint particle model must be derived. For the particle model in discrete time this is done in Section (5.2). In Section (5.3) it will be shown how the gradient can be determined from this adjoint model.

\section{The discrete time adjoint particle model}

\subsection{The discrete time particle model and the cost function}

For numerical simulations of a particle model the Langevin fluctuation equation must be discretized. Here this will be done by the Euler-scheme. This scheme is known to be of first order accuracy in weak sense (Kloeden and Platen, 1992). In this section the discrete time model is discussed. It will form the basis of the adjoint particle model that is derived in Section (5.2).

The following notation (sub and superscripts) is used in the discrete time model and the associated adjoint particle model:

$\mathrm{n}$ : discrete time; the time dependence of a variable is denoted by a superscript.

$\mathrm{k}$ : label for the $\mathrm{k}$-th particle; this label is denoted by a subscript.

$x$ : The subscript ' $x$ ' stands for the derivative of a function with respect to its spatial argument.

As a result we have for a track $\mathrm{X}_{\mathrm{k}}(\cdot)$ and a function $\mathrm{B}(\cdot \cdot)$ :

$$
\begin{aligned}
& X_{k}^{n}:=X_{k}(t), \quad t=n \Delta t \\
& B^{n}(x) ;=B(x, n \Delta t) ; B_{x}^{n}(\xi):=\left.\frac{\partial B(x, t)}{\partial x}\right|_{x=\xi, t-n \Delta t}
\end{aligned}
$$

Note that the spatial coordinate need not be discretized. The spatial dependencies will therefore be written in the 'function notation' as usual.

With these notations the discrete time particle model reads:

$$
\begin{aligned}
& \mathbf{n}=0: \mathbf{X}_{\mathbf{k}}^{0}=\xi_{\mathbf{k}} \quad \text { (initial condition) } \\
& \mathbf{n} \geq 1: \mathbf{X}_{\mathbf{k}}^{\mathbf{n}}=
\end{aligned}
$$

$$
X_{k}^{n-1}+\left\{\frac{Q^{n-1}\left(X_{k}^{n-1}\right)}{A^{n-1}\left(X_{k}^{n-1}\right)}+E_{x}^{n-1}\left(X_{k}^{n-1} \mid \alpha\right)+E^{n-1}\left(X_{k}^{n-1} \mid \alpha\right) \frac{A_{x}^{n-1}\left(X_{k}^{n-1}\right)}{A^{n-1}\left(X_{k}^{n-1}\right)}\right\} \Delta t+\sqrt{2 E^{n-1}\left(X_{k}^{n-1} \mid \alpha\right)} \Delta W_{k}^{n-1}
$$

The equation for the mass (13) can be integrated analytically:

$M_{k}(t)=\mu_{0} \cdot e^{-r_{k}(t)}$

Here $\Gamma_{k}(t)$, defined by equation (18), can in general not be solved analytically and is numerically approximated according to:

$$
\begin{aligned}
& n=0: \Gamma_{k}^{0}=0 \quad \text { (initial condition) } \\
& n \geq 1: \Gamma_{k}^{n}=\Gamma_{k}^{n-1}+\gamma^{n-1}\left(X_{k}^{n-1} \mid \beta\right) \Delta t
\end{aligned}
$$


With regard to the measurements it is assumed that these are available for a finite number of locations $x_{m}(m=1,2, \ldots, M)$, and on times that coincide with a discrete time $t^{n}=n \cdot \Delta t$. The measurement at $\left(x_{m}, t^{n}\right)$ is notated as $\hat{c}_{m}^{n}$, thus $\hat{c}_{m}:=\hat{c}\left(x_{m}, t^{n}\right)$. The model counterpart of a measurement concentration is:

$c_{m}^{n}=\frac{\mu_{0}}{A_{m}^{n}} \sum_{k=1}^{x} e^{-r_{k}^{n}} \phi^{n}\left(x_{m}-X_{k}^{n}\right)$

where $A_{m}^{n}:=A\left(x_{m}, t^{n}\right)$, see equation (19).

So the cost function for the discrete time particle model reads:

$J=\sum_{m=1}^{M} \sum_{n=1}^{N}\left(c_{m}^{a}-\hat{c}_{m}^{n}\right)^{2} \Psi_{m}^{n}$

Note that this formula suggests that the measured concentrations $\hat{\mathrm{c}} \mathbf{m}$ must be available for every combination of $x_{m}$ and $t^{n}$. In practice this will never be the case. To account for this sparseness the weight function must be set zero for all $(\mathrm{m}, \mathrm{n})$-combinations where a measured concentration $\hat{c}_{\mathrm{m}}^{\mathrm{n}}$ is absent.

\subsection{The discrete adjoint particle model}

The adjoint system is based on Lagrange's method for the optimization of functions subjected to constraints. Here the approach will be outlined for the discrete time particle model of Section (5.1).

In this case the cost function $\mathrm{J}(\mathrm{c}, \hat{\mathrm{c}})$ must be minimized with respect to the adjustable coefficients $\mathrm{E}(\cdot, \mid \alpha), \gamma(\because, \mid \beta)$ and $\mu_{0}$. Given the chain of dependencies \{coefficients\} $\rightarrow$ \{particle tracks\} $\rightarrow$ \{concentrations\} (see equation (21)), the model equations (23) form the constraints in this optimization problem.

The model equations are multiplied with Lagrange multipliers and added to the cost function $\mathrm{J}$. This leads to the following Lagrangian function L:

$$
\begin{aligned}
L= & \sum_{m=1}^{M} \sum_{n=0}^{N}\left(c_{m}^{n}-\hat{c}_{m}^{n}\right)^{2} \psi_{m}^{n}+ \\
& \sum_{m=1}^{M} \sum_{n=0}^{N} p_{m}^{n}\left\{c_{m}^{n}-\frac{\mu_{0}}{A_{m}^{n}} \sum_{k=1}^{K} e^{-r_{k}^{n}} \phi^{n}\left(x_{m}-X_{k}^{n}\right)\right\}+ \\
& \sum_{k=1}^{K} q_{k}^{0}\left(X_{k}^{0}-\xi_{k}\right)+\sum_{k=1}^{X} \sum_{n=1}^{N} q_{k}^{n}\left\{X_{k}^{n}-X_{k}^{n-1}-\left[\frac{Q^{n-1}\left(X_{k}^{n-1}\right)}{A^{n-1}\left(X_{k}^{n-1}\right)}+\right.\right. \\
& \left.\left.E_{x}^{n-1}\left(X_{k}^{n-1} \mid \alpha\right)+E^{n-1}\left(X_{k}^{n-1} \mid \alpha\right) \frac{A_{x}^{n-1}\left(X_{k}^{n-1}\right)}{A^{n-1}\left(X_{k}^{n-1}\right)}\right] \Delta t-\sqrt{2 E^{n-1}\left(X_{k}^{n-1} \mid \alpha\right) \Delta W_{k}^{n-1}}\right\}+ \\
& \sum_{k=1}^{K} r_{k}^{0} \Gamma_{k}^{0}+\sum_{k=1}^{K} \sum_{n=1}^{N} r_{k}^{0}\left\{\Gamma_{k}^{n}-\Gamma_{k}^{n-1}-\gamma^{n-1}\left(X_{k}^{n-1} \mid \beta\right) \Delta t\right\} .
\end{aligned}
$$


Next the sensitivity of $L$ is determined for the adjustable coefficients. For this purpose the parameters $\alpha, \beta$ and $\mu_{0}$ are perturbed by $\delta \alpha, \delta \beta, \delta \mu_{0}$ respectively and a first order variational analysis of $L$ is performed.

When the parameters $\alpha$ in the dispersion coefficient are varied with $\delta \alpha$, and the parameters $\beta$ in the decay coefficient with $\delta \beta$, the dispersion coefficient $E$ will vary with $\delta_{\alpha} E$ and the decay coefficient $\gamma$ with $\delta_{\beta} \gamma$. Moreover the particle tracks $X$ will be perturbed by $\delta \mathrm{X}$ (due to the particle model), the concentration by $\delta \mathrm{c}$ (since the concentration is a function of the particle tracks) and the cost function $J$ will finally be perturbed by $8 J$. For the Lagrange functional $\mathrm{L}$ this variational calculus leads to:

$$
\begin{aligned}
\delta L= & \sum_{m=1}^{M} \sum_{n=0}^{N} B_{m}^{n} \cdot \delta c_{m}^{n}+ \\
& \sum_{k=1}^{K} \sum_{n=0}^{N} F_{k}^{n} \cdot \delta X_{k}^{n}+ \\
& \sum_{k=1}^{K} \sum_{n=0}^{N} G_{k}^{n} \cdot \delta I_{k}^{n}+ \\
& \sum_{k=1}^{K} \sum_{n=0}^{N-1} H_{k}^{n} \cdot \delta_{\alpha} E^{n}\left(X_{k}^{n} \mid \alpha\right)+\sum_{k=1}^{K} \sum_{n=0}^{N-1} S_{k}^{n} \cdot \delta_{\alpha} E_{k}^{n}\left(X_{k}^{n} \mid \alpha\right)+ \\
& \sum_{k=1}^{K} \sum_{n=0}^{N-1} T_{k}^{n} \cdot \delta_{\beta} \gamma^{n}\left(X_{k}^{n} \mid \beta\right)+ \\
& \sum_{k=1}^{K} \sum_{m=1}^{M} \sum_{n=0}^{N} Y_{m, k}^{n} \cdot \delta \mu_{0} .
\end{aligned}
$$

The functional derivatives $B_{m}^{n}, F_{k}^{n}, G_{k}^{n}, H_{k}^{n}, S_{k}^{n}, T_{k}^{n}, Y_{m, k}^{n}$ are as follows:

$$
\begin{aligned}
& B_{m}^{n}=p_{m}^{n}+2\left(c_{m}^{n}-\hat{c}_{m}^{n}\right) \psi_{m}^{n} \\
& F_{k}^{n}=q_{k}^{n}-\left\{1+\left[\frac{A^{n}\left(X_{k}^{n}\right) Q_{x}^{n}\left(X_{k}^{n}\right)-Q^{n}\left(X_{k}^{n}\right) A_{x}^{n}\left(X_{k}^{n}\right)}{A^{2 n}\left(X_{k}^{n}\right)}+E_{x k}^{n}\left(X_{k}^{n} \mid \alpha\right)+.\right.\right. \\
& \left.\left.E_{x}^{n}\left(X_{k}^{n} \mid \alpha\right) \frac{A_{x}^{n}\left(X_{k}^{n}\right)}{A^{n}\left(X_{k}^{n}\right)}+E^{n}\left(X_{k}^{n} \mid \alpha\right) \frac{A^{n}\left(X_{k}^{n}\right) A_{x}^{n}\left(X_{k}^{n}\right)-A_{x}^{2 n}\left(X_{k}^{n}\right)}{A^{2 n}\left(X_{k}^{n}\right)}\right] \Delta t+\frac{E_{x}^{n}\left(X_{k}^{n} \mid \alpha\right)}{\sqrt{2 E^{n}\left(X_{k}^{n} \mid \alpha\right)}} \Delta W_{k}^{n}\right\} q_{k}^{n+1}+ \\
& \sum_{m=1}^{M} \frac{\mu_{0}}{A_{m}^{2}} e^{-r_{k}^{a}} \phi_{x}^{n}\left(x_{m}-X_{k}^{n}\right) p_{m}^{n}-\gamma_{x}^{n}\left(X_{k}^{n} \mid \beta\right) \Delta t r_{k}^{n+1} \\
& G_{k}^{n}=r_{k}^{n}-r_{k}^{n+1}+\sum_{m=1}^{M} \frac{\mu_{0}}{A_{m}^{n}} e^{-r_{k}^{k}} \phi^{n}\left(x_{m}-X_{k}^{n}\right) p_{m}^{n} \\
& H_{k}^{n}=-\left\{\frac{A_{x}^{n}\left(X_{k}^{n}\right)}{A^{n}\left(X_{k}^{n}\right)} \Delta t+\frac{\Delta W_{k}^{n}}{\sqrt{2 E^{n}\left(X_{k}^{n} \mid \alpha\right)}}\right\} q_{k}^{n+1}
\end{aligned}
$$


$S_{k}^{n}=-q_{k}^{n+1} \Delta t$

$\mathrm{T}_{\mathbf{k}}^{\mathrm{n}}=-\mathbf{r}_{\mathbf{k}}^{\mathrm{n}+1} \Delta \mathrm{t}$

$Y_{m, k}^{n}=-\frac{1}{A_{m}^{n}} e^{-r_{k}^{2}} \phi^{n}\left(x_{m}-X_{k}^{n}\right) p_{m}^{n}$

So far no assumptions have been made for the multipliers $p_{m}^{n}, q_{k}^{n}$ and $r_{k^{*}}^{n}$ In order to eliminate the part of $\delta \mathrm{L}$ that is not explicitly due to the parameter variations, the $p_{m}^{n}, q_{k}^{n}$ and $\mathbf{r}_{\mathrm{k}}^{\mathrm{n}}$ are chosen such that:

$\forall_{m, n, k}: B_{m}^{n}=0, F_{k}^{n}=0, G_{k}^{n}=0$

As a consequence the $p_{m}^{n}, q_{k}^{n}$ and $r_{k}^{n}$ satisfy the following equations:

$$
\begin{aligned}
& \mathbf{n}=\mathbf{N}: \mathbf{p}_{\mathrm{m}}^{\mathrm{n}}=-2\left(\mathrm{c}_{\mathrm{m}}^{\mathrm{n}}-\hat{\mathrm{c}}_{\mathrm{m}}^{\mathrm{m}}\right) \psi_{m}^{\mathrm{n}}, \mathrm{q}_{\mathrm{k}}^{\mathrm{n}}=0, \mathrm{r}_{\mathrm{k}}^{\mathrm{n}}=0 \\
& n<N: p_{m}^{n}=-2\left(c_{m}^{n}-\hat{c}_{m}^{n}\right) \Psi_{m}^{n} \\
& \mathbf{q}_{k}^{n}=\left\{1+\left[\frac{A^{n}\left(X_{k}^{n}\right) Q_{x}^{n}\left(X_{k}^{n}\right)-Q^{n}\left(X_{k}^{n}\right) A_{x}^{n}\left(X_{k}^{n}\right)}{A^{2 n}\left(X_{k}^{n}\right)}+E_{x x}^{n}\left(X_{k}^{n} \mid \alpha\right)+\right.\right. \\
& \left.\left.E_{x}^{n}\left(X_{k}^{n} \mid \alpha\right) \frac{A_{x}^{n}\left(X_{k}^{n}\right)}{A^{n}\left(X_{k}^{n}\right)}+E^{n}\left(X_{k}^{n} \mid \alpha\right) \frac{A^{n}\left(X_{k}^{n}\right) A_{x k}^{n}\left(X_{k}^{n}\right)-A_{x}^{2 n}\left(X_{k}^{n}\right)}{A^{2 n}\left(X_{k}^{n}\right)}\right] \Delta t-\frac{E_{x}^{n}\left(X_{k}^{n} \mid \alpha\right)}{\sqrt{2 E^{n}\left(X_{k}^{n} \mid \alpha\right)}} \Delta W_{k}^{n}\right\} q_{k}^{n+1} \\
& \sum_{m=1}^{M} \frac{\mu_{0}}{A_{m}^{n}} e^{-r_{x}^{n}} \phi_{x}^{n}\left(x_{m}-X_{k}^{n}\right) p_{m}^{n}+\gamma_{x}^{n}\left(X_{k}^{n} \mid \beta\right) \Delta t x_{k}^{n+1} \\
& \mathbf{r}_{k}^{\mathrm{n}}=\mathbf{t}_{\mathbf{k}}^{\mathrm{n}+1}-\sum_{m=1}^{M} \frac{H_{0}}{A_{m}^{\mathrm{n}}} \mathbf{e}^{-r_{\mathbf{k}}^{\mathrm{n}}} \phi^{\mathrm{n}}\left(x_{m}-X_{k}^{\mathrm{n}}\right) p_{m}^{n}
\end{aligned}
$$

These dynamical equations form the adjoint particle model. This model has the same properties as the adjoint models known for finite difference models (see e.g. Chavent, 1980; Merckx, 1987; van den Boogaard, 1988; ten Brummelhuis, 1992). The adjoint model is a linear system and must be solved in reversed time order. It is not homogeneous since it is forced by the residuals of model concentrations and observed concentrations. Moreover the adjoint model is independent of the parameterization of the adjustable model coefficients. In computer applications this allows a great flexibility in the parameterization of these coefficients. The most important advantage of the adjoint model is however its efficiency in the calculation of the gradient of the cost function. In Section 5.3 it is shown how this must be done in the present case. 


\subsection{The gradient of the cost function}

Since the $\left\{c_{m}^{n}, X_{k}^{n}, \Gamma_{k}^{n} \mid m=1,2, \ldots, M ; k=1,2, . . K ; n=0,1, \ldots, N\right\}$ satisfy the model equations (see equation (23)), and the $\left\{p_{m}^{n}, q_{k}^{n}, r_{k}^{n} \mid m=1,2, \ldots, M ; k=1,2, . K ; n=0,1\right.$, ..., N\} satisfy the adjoint model equations, (see equation (29)), it follows that

$$
\begin{aligned}
\delta L=\delta J= & \sum_{k=1}^{K} \sum_{n=0}^{N-1} H_{k}^{n} \cdot \delta_{\alpha} E^{n}\left(X_{k}^{n} \mid \alpha\right)+\sum_{k=1}^{K} \sum_{n=0}^{N-1} S_{k}^{n} \cdot \delta_{\alpha} E_{\alpha}^{n}\left(X_{k}^{n} \mid \alpha\right)+ \\
& \sum_{k=1}^{K} \sum_{n=0}^{N-1} T_{k}^{n} \cdot \delta \gamma^{n}\left(X_{k}^{n} \mid \beta\right)+ \\
& \sum_{k=1}^{K} \sum_{m=1}^{M} \sum_{n=0}^{N} Y_{m, k}^{n} \cdot \delta \mu_{0}
\end{aligned}
$$

The functional derivatives $H_{k}^{n}, S_{k}^{n}, T_{k}^{n}$ and $Y_{m, k}^{n}$ are defined by equations (27d-g).

From these results a 'simple' expression for the gradient of the cost function can be derived. By virtue of

$$
\delta_{\alpha} E^{n}\left(X_{k}^{n} \mid \alpha\right)=\sum_{i=1}^{A} \frac{\partial E^{n}\left(X_{k}^{n} \mid \alpha\right)}{\partial \alpha_{i}} \delta \alpha_{i} \text { and } \delta_{\alpha} E_{x}^{n}\left(X_{k}^{n} \mid \alpha\right)=\sum_{i=1}^{A} \frac{\partial E_{x}^{n}\left(X_{k}^{n} \mid \alpha\right)}{\partial \alpha_{i}} \delta \alpha_{1}
$$

it is found that

$$
\frac{\partial J}{\partial \alpha_{i}}=-\sum_{k=1}^{K} \sum_{n=0}^{N-1} q_{k}^{n+1}\left[\left\{\frac{A_{k}^{n}\left(X_{k}^{n}\right)}{A^{n}\left(X_{k}^{n}\right)} \Delta t+\frac{\Delta W_{k}^{n}}{\sqrt{2 E^{n}\left(X_{k}^{n} \mid \alpha\right)}}\right\} \frac{\partial E^{n}\left(X_{k}^{n} \mid \alpha\right)}{\partial \alpha_{i}}+\Delta t \frac{\partial E_{z}^{n}\left(X_{k}^{n} \mid \alpha\right)}{\partial \alpha_{i}}\right]
$$

Similarly, the derivative of the cost function with respect to the $i$-th parameter $\beta_{i}$ in the parameterization of the decay coefficient is given by

$$
\frac{\partial J}{\partial \beta_{1}}=-\Delta t \sum_{k=1}^{R} \sum_{n=0}^{N-1} \mathbf{r}_{k}^{n+1} \frac{\partial \gamma^{n}\left(X_{k}^{n} \mid \beta\right)}{\partial \beta_{i}}
$$

For the derivative of the cost function with respect to the mass of a particle it is found that:

$$
\frac{\partial J}{\partial \mu_{0}}=-\sum_{m=1}^{M} \sum_{n=0}^{N} \frac{p_{m}^{n}}{A_{m}^{n}} \sum_{k=1}^{K} e^{-r_{k}^{n}} \phi^{n}\left(x_{m}-X_{k}^{n}\right)
$$

\section{Applications}

On the basis of the theory derived in the preceding sections, a computer program was developed for the identification of parameters in 1D-particle models. The main components of this program are a particle model, the adjoint particle model, and a Quasi-Newton gradient method for the updating of uncertain parameters, The program is written in standard FORTRAN-77 and runs on a PC-80386 computer. The implementation of the particle model, its adjoint, and the computation of the gradient of the cost function were extensively 
tested and verified.

In this section two applications are presented. In the first example artificial data are used. These data are generated with a finite difference model. Apart from the performance of the proposed calibration technique, the consistency of the particle model with finite difference models is verified. In the second example, a "real life case" is considered and the calibration technique is applied to water quality data that were obtained from the river Rhine during the Sandoz calamity of November 1986.

\subsection{Application to artificial data}

In this example an imaginary estuary is considered with a tidal flow described by:

$$
Q(x, t)=Q_{1} \cdot \cos (\omega \cdot t-k \cdot x)
$$

and

$A(x, t)=A_{0}+A_{1} \cdot \cos (\omega \cdot t-k \cdot x)$.

Since the discharge and cross-section must satisfy the continuity equation,

$$
\frac{\partial A}{\partial t}+\frac{\partial Q}{\partial x}=0
$$

the constants $Q_{1}$ and $A_{1}$ are related as follows:

$$
A_{1}=\frac{k}{\omega} Q_{1}=\frac{Q_{1}}{c}
$$

$\mathrm{c}$ is the phase velocity which can be calculated from the depth $\mathrm{H}$ of the estuary according to $\mathrm{c}^{2}=\mathrm{g} \cdot \mathrm{H}$.

In this estuary a solute is released whose spread is described by the advection-diffusion equation (14). For the decay coefficient $\gamma(\because)$ and dispersion coefficient $\mathrm{E}(\because)$ the following parameterizations were used:

$$
\gamma(\mathbf{x}, \mathbf{t} \mid \boldsymbol{\beta})=\beta
$$

$$
E(x, t \mid \alpha)=\exp \left[\alpha_{1}+\alpha_{2} \cdot \cos (\omega t-k \cdot x)\right]
$$

The advection diffusion was solved with a finite-difference numerical model (based on a Crank-Nicholson scheme; see e.g. Press et al., 1989). In this computation the following values were used for the flow parameters: $Q_{1}=500 \mathrm{~m}^{3} / \mathrm{s}, A_{0}=1000 \mathrm{~m}^{2}, \mathrm{H}=4 \mathrm{~m}$, and radian frequency $\omega=2 \pi / T$ with period $T=12$ hours. The adjustable parameters in the dispersion coefficient and the decay coefficient were chosen as follows: $\alpha_{1}=0.5, \alpha_{2}=-2.5$ and $\beta=$ $0.75 /$ day ("true values").

For the initial distribution of the concentration a Gaussian profile was used with peak at $x=0$ and spread $a=250 \mathrm{~m}$ (no "true peak", i.e. $\sigma=0$, was taken since finite difference 
models cannot appropriately deal with such initial conditions). The Gaussian profile was normalized such that the total initial mass $M$ of the solute is $1000 \mathrm{~kg}$. In the finite difference model an equidistant grid was used $(\Delta x=100 \mathrm{~m})$ and 4 periods were simulated (1440 time steps with $\Delta \mathrm{t}=2$ minutes).

From the concentrations computed with the finite difference model, a set of "observed concentrations" $\left\{\hat{\mathrm{c}}\left(\mathrm{x}_{\mathrm{m}}, \mathrm{t}_{\mathrm{m}}\right) \mid \mathrm{m}=1,2, \ldots, \mathrm{M}\right\}$ was generated. To imitate practical conditions where observation sets are usually very sparse (both in time and in space), samples $\hat{c}(x, t)$ are taken from no more than two locations $\left(x_{1}=0\right.$ and $\left.x_{2}=2.5 \mathrm{~km}\right)$, and at time points at least 4 hours after the calamity (times $4 \mathrm{~h}, 6 \mathrm{~h}, 8 \mathrm{~h}, \ldots, 48 \mathrm{~h}$ ). This leads to a set of 46 observed samples.

These observations were used by the particle model to estimate the adjustable parameters in the dispersion coefficient $\left(\alpha_{1}, \alpha_{2}\right)$, the decay coefficient $(\beta)$ and the initial mass of a particle $\left(\mu_{0}\right)$. In this calibration 1000 particles were used. As initial guess $\mu_{0}=0.5 \mathrm{~kg}, \gamma=$ $0, \alpha_{1}=2.5$ and $\alpha_{2}=0$ were taken.

After 20 iterations involving 24 evaluations of the both the particle model and its adjoint, the following estimations were found for the adjustable parameters: $\mu_{0}=1.091 \mathrm{~kg}, \beta=$ $0.768 /$ day, $\alpha_{1}=0.411$ and $\alpha_{2}=-2.60$. The cost function was reduced from 0.257 to $0.125 \cdot 10^{-2}$ $\left(\mathrm{gr} / \mathrm{m}^{3}\right)^{2}$.

The iteration process is illustrated in the Figures 1a-c. Figure 1a shows the cost function and the $\mathrm{L}_{2}$-norm of the gradient as function of the iteration step. Figure $1 \mathrm{~b}$ gives the estimations of the adjustable parameters as function of the iteration. For four positions $\mathbf{x}_{\mathrm{i}}$, Figure 1c compares the following four concentration time series $t \rightarrow c\left(x_{j}, t\right)$ : (i) the "true" concentrations according to the finite difference model, (ii) the subset of (i) used as observations, (iii) the concentrations according to the particle model with the initial guess of the uncertain parameters, and (iv) the concentrations of the calibrated particle model.

It is seen that the "true" concentrations and the concentrations of the calibrated particle model are only marginally different. Although not shown here, a very close agreement was found with the concentrations of the particle model with the true parameters as well (in fact, for the true values of the parameters the cost function was $0.135 \cdot 10^{-2}$, which is only slightly greater than the minimum of the cost function).

From these results it is concluded that (i) the calibration technique performs well, and (ii), the accuracy the particle model compared well with the accuracy of the finite difference model.

\subsection{Application to river Rhine data}

During the extinguish of a fire at the chemical works of Sandoz at Basel in Switzerland (in the night of October 31 to November 1,1986 ) a large amount of toxic solutes were washed into the river Rhine. In the days that followed, these solutes moved downstream through the German part of the river reaching the German-Dutch border at Lobith after approximately eight days.

For one of the solutes (disulphoton) concentration measurements are available at three locations: Mainz (498 kin), Bad Honnef $(640 \mathrm{~km})$ and Lobith $(862 \mathrm{~km})$. The numbers within the parenthesis denote the distance of these locations to Konstanz (considered as the offspring of the river Rhine; the distance of Basel to Konstanz is $170 \mathrm{~km}$ ).

Apart from the disulphoton measurements, a large number of waterlevel registrations were available for several stations. By means of $h-T$ tables (relating waterlevels and duration times) and h-Q tables (relating water levels and discharges) it was possible to derive the discharges and cross sections along the solute's trajectory. These discharges and cross section areas varied both in time and along the flow direction.

The computer model was applied to the data available for this calamity. In this way the initially released mass of the solute, the decay coefficient, and the dispersion coefficient were 

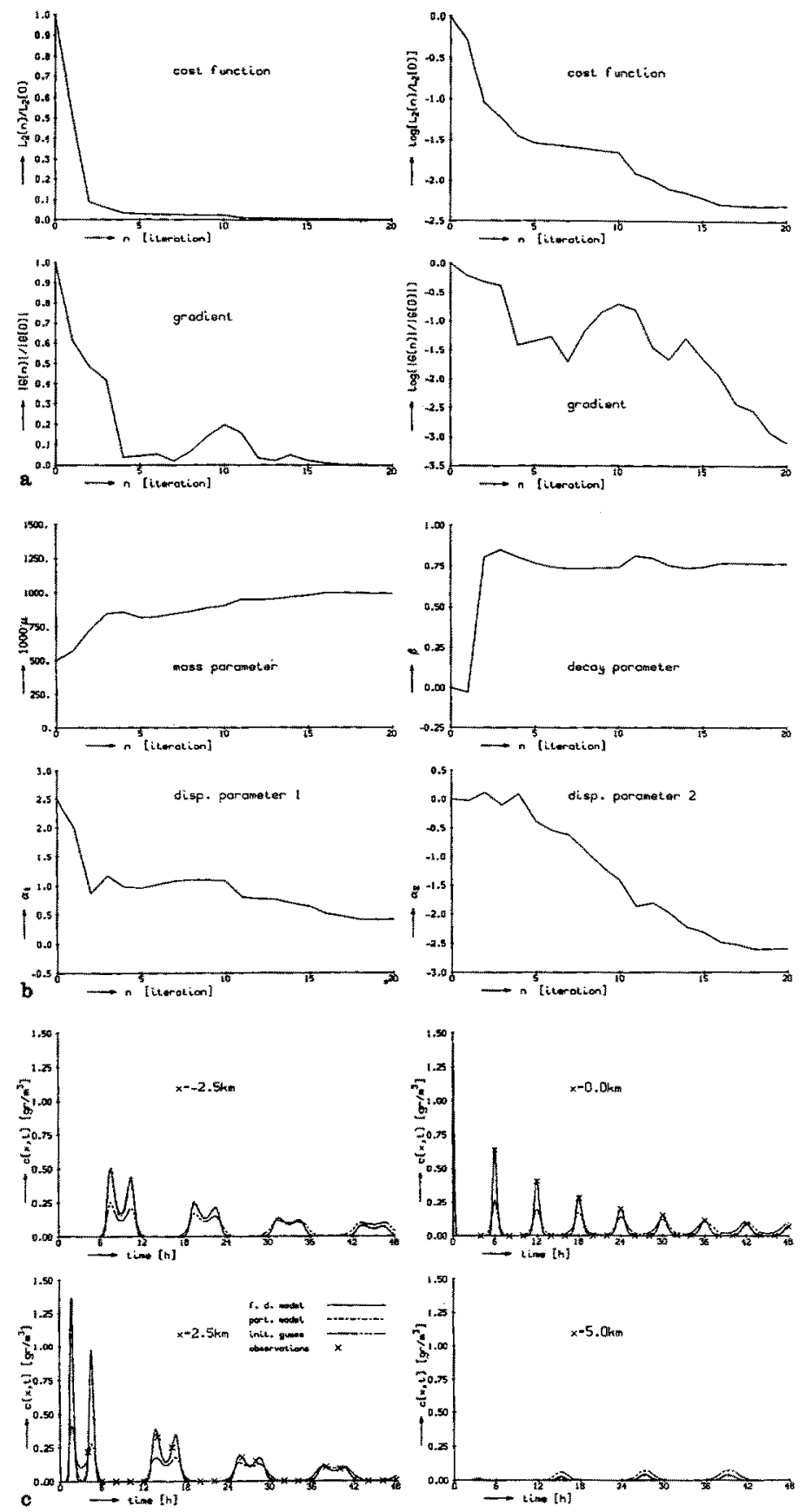

Figure 1. (a) Application of calibration technique to artificial data. Cost function and gradient as function of the iteration step. (b) Application of calibration technique to artificial data. Adjustable parameters as function of the iteration step. (c) Application of calibration technique to artificial data. Time series of concentrations. For further explanation see Section 6.1. 
estimated. The decay coefficient and the dispersion coefficient were parameterized by the constants $\beta$ and $\alpha$ respectively: $\gamma(x, t \mid \beta)=\beta$ and $E(x, t \mid \alpha)=\alpha$.

As intial guess, estimations of the parameters were used that were obtained in earlier studies on this calamity: $\beta=0.25 /$ day, $\alpha=400 \mathrm{~m}^{2} / \mathrm{s}$ and total initial mass $\mathrm{M}_{0}=6768 \mathrm{~kg}$. In the particle model 1000 particles were used (leading to an initial guess $\mu_{0}=6.768 \mathrm{~kg}$ for a particles initial mass), the time step $\Delta t=0.05$ days, and 12 days ( $=240$ time steps) were simulated. After 3 iterations (involving 10 evaluations of the particle model and its adjoint) the following estimations were found for the parameters: $\mu_{0}=6.90 \mathrm{~kg}$ (thus $\mathrm{M}_{0}=6900 \mathrm{~kg}$ ), $\beta=0.228 /$ day and $\alpha=299 \mathrm{~m}^{2} / \mathrm{s}$. The cost function was reduced from 86.45 to 28.89 $(\mu \mathrm{gr} / \mathrm{liter})^{2}$.

For the three locations where observations were available, the measured disulphoton concentrations ( $x$ samples). and the concentrations according to the model with the initial guess of the parameters (dashed curves), and the calibrated particle model (solid curves) are shown in Figure 2a. For Mainz and Bad Honnef the calibrated concentrations agree well with the observations. For Lobith the samples in the 'tail' ( $t \geq 9 \mathrm{~d})$ are somehow less accurately reproduced by the model. This may be partly due to model deficiencies: it is known that a one dimensional projection of three or two dimensional solute transport is only valid under special circumstances, see e.g. Bhattacharya and Waymire (1990). Moreover, in the present model it is not accounted for riparian lands along the German part of the river. In these so called dead zones a part of the solute's mass can be temporarily stored. In downstream stations this effect is noticed in the form of enhanced tails in the concentration time series.

Despite these criticisms, one dimensional transport models of the form of equation (14) are used very often in practice and this initiated the development of the present calibration technique.

For three time points Figure $2 \mathrm{~b}$ shows the spatial distribution of the calibrated concentration along the river.

\section{Conclusions and discussion}

In this paper a technique has been developed for the identification of parameters in particle models. This technique is based on adjoint modelling. The advantages of this method known from earlier applications in finite difference models hold here as well. These advantages are the computational efficiency (for the calculation of the gradient of a cost function) and flexibility (the adjoint particle model is independent of the parameterization of the adjustable model coefficients).

The calibration technique was applied to artificial data and to field data obtained during the Sandoz calamity. These applications demonstrate the feasibility of the method in practice. It turned out that it is possible to estimate simultaneously the mass of the dissolved matter, the decay coefficient and the dispersion coefficient.

The technique has been developed and applied for one dimensional particle models. It can however straightforwardly be generalized to random walk models in higher dimensions. Moreover it is possible to incorporate additional physical/chemical processes or alternative constraints such as density effects, boundary conditions (e.g. reflecting or absorbing barriers), or particle interaction dynamics. 

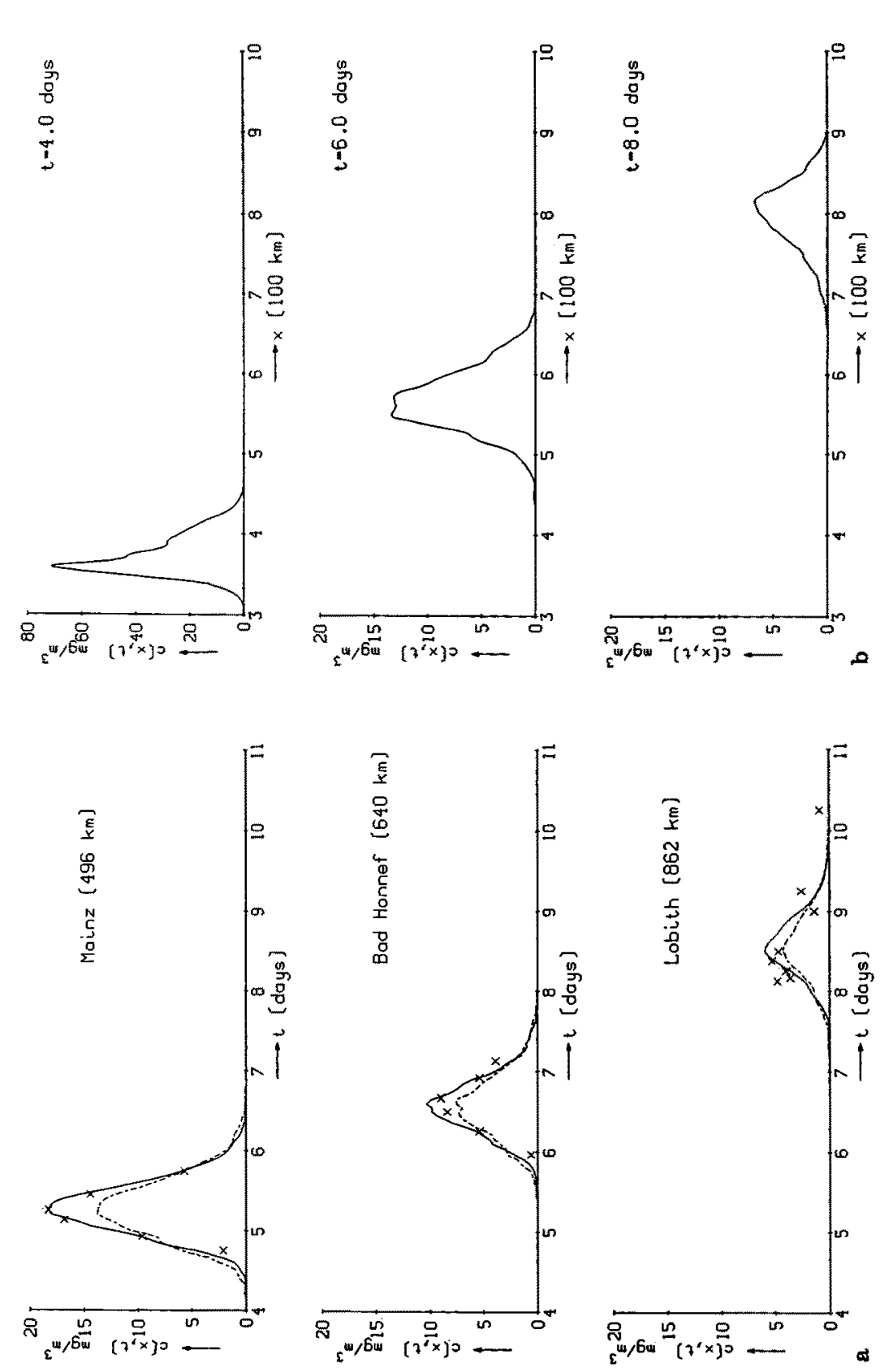

Figure 2. (a) Application of calibration technique to Sandoz calamity. Concentration time series $t \rightarrow c(x, t)$ tbr three stations along the river Rhine for the optimized model. For further explanation see Section 6.2. (b) Application of calibration technique to Sandoz calamity. Concentration profiles $x \rightarrow c(x, t)$ along the river Rhine for three time points. For further explanation see Section 6.2 . 


\section{References}

Bhattacharya, R. N.; Waymire, E. C. 1990: Stochastic Processes with Applications. Wiley Series in Probability and Mathematical Statistics. Wiley, New York

Chavent, G. 1980: Identification of distributed parameter systems: about the output least square method, its implementation, and identifiability. In: Iserman R. (ed.) Proc. 5th IFAC Symposium on Identification and System Parameter Estimation, Vol I, pp 85-97. New York: Pergamon Press

Heemink, A. W. 1986: Storm surge prediction using Kalman filtering. Ph.D. thesis, Twente University of Technology, The Netherlands

Heemink, A. W. 1990: Stochastic modelling of dispersion in shallow water. Stochastic Hydrology and Hydraulics, No. 4., pp. 161-174

Kloeden, P. E.; Platen, E. 1992: Numerical solution of stochastic differential equations. Applications of Mathematics 23, Springer-Verlag, New York

Merckx, C. 1987; Identification of a spatially varying parameter in a time-periodic parabolic system. Int. J. Control 46, No. 2, pp. 699-708

Middleton, D. 1960: An introduction to statistical communication theory. New York: McGraw Hill

Press, W. H.; Flannery, B. P.; Teukolsky, S. A.; Vetterling, W. T. 1989: Numerical recipes. The Art of Scientific Computing. Cambridge: Cambridge University Press

van den Boogaard, H. F. P. 1988: Calibration of mathematical models by optimal control. Delft: Delft Hydraulics Report Z62/10, Z107/04 (in Dutch)

van Kampen, N. G. 1981a: Stochastic processes in physics and chemistry. Amsterdam: North Holland

van Kampen, N. G. 1981b: Ito versus Stratonovich. Journal of Statistical Physics 24, No. 1

Stratonovich, R. L. 1963: Topics in the theory of random noise. Vol. I.. New York: Gordon and Breach, New York

ten Brummelhuis, P. G. J. 1992: Parameter estimation in tidal models with uncertain boundary conditions. Ph.D. Thesis, Twente University of Technology, The Netherlands

Uffink, G. J. M. 1990: Analysis of dispersion by the random walk method. Ph.D. Thesis, Delft University of Technology, The Netherlands 


\section{Appendix A. Calculation of concentrations from particle tracks}

Let $\left\{X_{1}(t), X_{2}(t), X_{3}(t), \ldots, X_{K}(t)\right\}$ denote the random positions of $K$ particles at time $t$. Each particle represents a unit of mass $\mu_{0}$, and as a result the mass density distribution $\mu_{0}(\cdot ;)$ satisfies,

$\mu(x, t)=\mu_{0} \sum_{k=1}^{X} \delta\left(x-X_{k}(t)\right)$

Although not necessary, it is assumed that all particles have equal mass.

According to equation (Al) the mass density distribution is a series of infinitely narrow peaks and it is a discontinuous and extremely nonsmooth function. In practice, as time evolves, the solute's mass distribution is much more continuous and smooth, however. Therefore the particle's mass is spread out over a small interval surrounding its position $\mathrm{X}_{\mathrm{k}}(\mathrm{t})$. This means that each peak is replaced by a point spread function $\phi(\cdot)$ centered at $\mathrm{X}_{\mathrm{k}}(\mathrm{t})$. This can be seen as a spatial filtering of the right hand side of equation (Al) leading to:

$\mu(\mathrm{x}, \mathrm{t})=\mu_{0} \sum_{\mathrm{k}=1}^{\mathbf{K}} \phi\left(\mathrm{x}-\mathrm{X}_{\mathrm{k}}(\mathrm{t})\right)$ where $\int_{--}^{-} \phi(\xi) \mathrm{d} \xi=1$

By virtue of equation $(8), \mu(x, t)=A(x, t) \cdot c(x, t)$, and the mass density function can be translated into a concentration $c(x, t)$ :

$c(x, t)=\frac{\mu_{0}}{A(x, t)} \sum_{k=1}^{K} \phi\left(x-X_{k}(t)\right)$

A Gaussian profile is taken here for the point spread function $\phi(\cdot)$ and as a result

$\phi(x)=\frac{1}{\sigma \sqrt{2 \pi}} \exp -\frac{1}{2}\left(\frac{x}{\sigma}\right)^{2}$

In simulations the width $\sigma$ must depend on time $t$ since the particles are distributed more sparsely as time evolves (due to the increase of the variance of the particle's random position). Therefore:

$\phi(x, t)=\frac{1}{\sigma(t) \sqrt{2 \pi}} \exp -\frac{1}{2}\left(\frac{x}{\sigma(t)}\right)^{2}$

It can be derived that $\sigma(t)$ must be proportional to $\sqrt{2 \int_{0}^{t} E(s) d s}$ and proportional to $\frac{1}{\sqrt[5]{K}}$ (as $\mathrm{K} \rightarrow \infty$ ) where $\mathrm{K}$ is the number of particles.

In case the solute is subjected to decay, the point spread function must be multiplied by the exponent of the decay coefficient integrated along the particle's trajectory. This can be seen from analytical integration of the differential equation for the particle's mass: 
$d M(t)=-\gamma(\mathbf{X}, t) \cdot M(t) \cdot d t \rightarrow M(t)=\mu_{0} \cdot \exp \left(-\int_{v}^{t} \gamma(\mathbf{X}(s), s) d s\right)$

This leads to

$c(x, t)=\frac{\mu_{0}}{A(x, t)} \sum_{k=1}^{K} e^{-T_{k}(t)} \phi\left(x-X_{k}(t), t\right)$

where $\Gamma_{k}(\cdot)$ is defined by:

$\Gamma_{k}(t)=\int_{b}^{t} \gamma\left(\mathbf{X}_{k}(s), s\right) d s$. 


\section{Appendix B. Particle models for decaying solutes}

In Section 3, it was shown that the transport of non-decaying solutes can be modelled by a random walk of a large number of particles. The fluctuation equation (11) describes a particle's position $\mathbf{X}(\cdot)$ as function of time.

For solutes subjected to decay this model must be extended with a second fluctuation equation that describes the time evolution of the particle's mass $\mathbf{M}(\cdot)$. It is common use to model the mass decay $\Delta \mathrm{M}(\mathrm{t})$ within a time interval $(\mathrm{t}, \mathrm{t}+\Delta \mathrm{t})$ with a term propoftional to $\Delta t$, and proportional to the actual mass $M(t)$. Together with equation (11) this leads to the following two coupled stochastic differential equations:

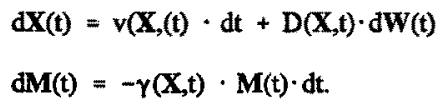

Note that $\mathrm{t} \rightarrow(\mathbf{X}(\mathrm{t}), \mathbf{M}(\mathrm{t}))^{\mathrm{T}}$ forms a bivariate Markov process. In the remainder of this section it is shown that these equations form a particle model that is consistent with the non-conservative transport equation (14).

The probability density function $\mathrm{f}\left({ }^{\circ},\right)$, defined by

$f(x, m ; t):=\lim _{\Delta x+0, \Delta m b 0} \frac{P[x \leq X(t)<x+\Delta x \text { and } m \leq M(t)<m+\Delta m]}{\Delta x \cdot \Delta m}$

describes the distribution of the bivariate stochastic process $t \rightarrow(\mathbf{X}(t), \mathbf{M}(t))^{\mathrm{T}}$. It can be shown that $\mathrm{f}(, ;)$ satisfies the following Fokker-Planck equation:

$$
\frac{\partial}{\partial t} f(x, m ; t)=-\frac{\partial}{\partial x}[v(x, t) f(x, m ; t)]+\frac{\partial}{\partial m}[\gamma(x, t) m \cdot f(x, m ; t)]+\frac{1}{2} \frac{\partial^{2}}{\partial x^{2}}\left[D^{2}(x, t) f(x, m ; t)\right]
$$

See e.g. Middleton (1960).

For particles that are at position $\mathrm{x}$ at time $\mathrm{t}$, the expectation of their mass is:

$$
\langle m(x, t)\rangle=E[M(t) \mid X(t)=x]=\int m \cdot f(x, m ; t) d t
$$

Multiplication of each term in equation (B2) with $\mathrm{m} \cdot \mathrm{dm}$, followed by integration over the $\mathrm{m}$-domain, leads to the following partial differential equation for $\langle\mathrm{m}(\because ;)\rangle$ :

$$
\frac{\partial}{\partial t}\{m(x, t)\rangle=-\frac{\partial}{\partial x}[v(x, t)\langle m(x, t))]-\gamma(x, t) \cdot\{m(x, t)\rangle+\frac{1}{2} \frac{\partial^{2}}{\partial x^{2}}\left[D^{2}(x, t)(m(x, t)\rangle\right] .
$$

Since the mass-density $<m(x, t)>$, cross-section $A(x, t)$ and concentration $c(x, t)$ are related according to $\langle\mathrm{m}(\mathrm{x}, \mathrm{t})\rangle=\mathrm{A}(\mathrm{x}, \mathrm{t}) \cdot \mathrm{c}(\mathrm{x}, \mathrm{t})$, the concentration satisfies:

$$
\frac{\partial}{\partial t}[A(x, t) \cdot c(x, t)]=-\frac{\partial}{\partial x}\left[v(x, t) \cdot A(x, t) \cdot c(x, t)+\frac{1}{2} \frac{\partial^{2}}{\partial x^{2}}\left[D^{2}(x, t) \cdot A(x, t) \cdot c(x, t)\right]-[\gamma(x, t) \cdot A(x, t)\right.
$$


Substitution of the drift $v(", ;)$ and diffusion coefficient $D(\because ;)$, prescribed by equation (12), leads to the non-conservative transport equation (14). 